\title{
Effect of Zinc and Iron on Growth and Yield of GrandNain Banana Cultivar in Sandy Soil
}

Abd Rabou, F. A 1

\section{ABSTRACT}

A field study was carried out during 2008/20092009/2010 in Grand Nain (the plantlets developed from local meristems, superior selected from Grand Nain Banana $c v$.) grown in private orchard at El khtatba, Monofia Governorate, during two successive seasons to determine the $\mathrm{Zn}$ and $\mathrm{Fe}$ requirements. The plants were received different rates of $\mathrm{Zn}$ and $\mathrm{Fe}$ fertigation. The rates per plants were $((0.0,5.4,10.8$ and $21.6 \mathrm{gm} / \mathrm{plant})$ in the form of $\mathrm{ZnS}_{\mathrm{O} 4}$ and $(\mathbf{0 . 0}, 9.0,18.0,36.0 \mathrm{gm} / \mathrm{plant})$ actual $\mathrm{Fe}$ (EDTA). All considered $\mathrm{Zn}$ and $\mathrm{Fe}$ rates were applied every month from April till October and were added as solution by fertigation. Results show that, the increasing rates of each fertilizer ( $\mathrm{Zn}$ and $\mathrm{Fe}$ ) increased plant height, pseudostem girth, leaves number, leaf area and reduced the number of days taken shooting till flowering. Znfertigation had greatest effect on vegetative growth, time to shooting and harvesting. The uppermost $\mathrm{Zn}$ level (21.6g./plant) resulted in progressive increase in growth characteristics and yield. Application of (36.0g.Fe/plant) results in a significant increase in growth finger characteristics . Increasing $\mathrm{Zn}$ rates raised leaf $\mathrm{N}, \mathrm{P}, \mathrm{K}, \mathrm{Zn}$ and Fe. Increasing rate of Fe-fertigation increased leaf $\mathbf{N}$, $\mathrm{P}, \mathrm{K}, \mathrm{Zn}$ and $\mathrm{Fe}$.
\end{abstract}

Application of 21.6g. $\mathrm{Zn} / \mathrm{plant}$ and 36.0g.Fe/plant is monthly from April to October recommended for GrandNain banana in sandy soil.

Keyword: Banana fertigation, fertigation by $\mathrm{Zn}$ and Fe, Micro-nutrition

\section{INTRODUCTION}

Banana (Musa spp.) is a tropical plant and was considered as one of the most popular and favorite fruits in the world since, fruits have excellent flavor, nice taste and high nutritional value, in addition to some miscellaneous uses and a number of minor edible products.

In Egypt, banana production was not sufficient to cover the demands of local Egyptian markets, the latest statistics of the ministry of Agriculture in 2009 referred that the area occupied by banana plants in the Delta regions and Nile valley was estimated by 62236 feddans, and generally produced 1122698 tons.

From the botanical view, banana plant was considered as a herbaceous mesophytic plant, with fast growth rate in nursery and field.

Although only minor quantities of micro-nutrients are needed, they are indispensable to the vegetative growth and yield of banana (Twyford, 1967). Very little work has been carried out on trace elements requirements of banana plants. However the importance of these trace elements on vegetative growth and production of banana had been recorded for zinc (Jordine, 1962 and Nason, 1950) and Iron (Ziv, 1954), (Abdel- Kader,1986, Hamam, 1988, Abdel-Kader et al., 1992, Sallam et al.,2002 and Ghanta and Mitra 1993) applied mixture of micro-nutrients as foliar spray ( $\mathrm{Zn}$, $\mathrm{Cu}, \mathrm{B}$ and $\mathrm{Mg}$ ) beside the common programmer of $\mathrm{N}$, $\mathrm{P}$ and $\mathrm{K}$ fertilizations applied to Hindy banana plants increased the number of green leaves, improved bunch shooting and greatly increased bunch weight in plants (Moreira et al., 2007). Zinc is absorbed by the roots and quickly transported to the aerial part. It is partially mobile within the plant and its transport occurs passively through transpiration flow (Epstein and Bloom, 2005). Nevertheless, the transport mechanisms of sap in the xylem are subject of considerable debate (Longnecker and Robson, 1993). Zinc is known to have an important role as a metal component of enzymes or as a functional structural or regulatory cofactor of a large enzyme (Werner, 1992). Zinc accelerates auxin synthesis or protects auxin from oxidative destruction (Cakmak, 1988). Zinc deficiency reduced the distance between hands and giving them bunches with a compact appearance (Brown et al., 1993 and Borges et al., 2006). Swidan (1972) noticed that the differentiation of inflorescences in banana plants cv. Hindi is positively correlated with the total leaf area per plant.

The objective of this study was to determine the optimum amount of zinc sulfate and iron (EDTA) added by fertigation around the year/plant to obtain high yield, good quality and to reduce the cost of fertilizers of Grand Nain banana plants in the newly reclaimed sandy soil under drip irrigation system.

\section{MATERIALS AND METHODS}

This experiment was carried out during two successive seasons of 2008/2009 and 2009/2010 on GrandNain banana cultivar growing in private orchard at El khtatba, Monofia Governorate Egypt. The physical and chemical analysis of sandy soil are presented in Table (1).

Plants were planted at $3.5 \times 3 \mathrm{~m}$ apart in March 2007. Suckers nearly similar in vigour and growth.

${ }^{1}$ Horticulture Research Institute, Tropical Fruits Research Department. Received May16, 2011, Accepted June 14, 2011 
Table 1. Soil characteristics of the banana plantation at the start of experiment (a): Physical analysis

\begin{tabular}{|c|c|c|c|c|c|c|c|c|c|}
\hline \multirow{4}{*}{$\begin{array}{r}\text { Depth } \\
\mathrm{cm} \\
\end{array}$} & \multirow{2}{*}{\multicolumn{7}{|c|}{ Mechanical analysis \% }} & \multirow{4}{*}{$\begin{array}{c}\text { CaCo3 } \\
\%\end{array}$} & \multirow{4}{*}{$\begin{array}{c}\text { Textural } \\
\text { class }\end{array}$} \\
\hline & & & & & & & & & \\
\hline & \multirow{2}{*}{\multicolumn{2}{|c|}{$\begin{array}{c}\text { Course } \\
\text { sand } \\
\%\end{array}$}} & \multirow{2}{*}{$\begin{array}{c}\text { Fine } \\
\text { sand } \\
\% \\
\end{array}$} & \multirow{2}{*}{$\begin{array}{c}\text { Silt } \\
\%\end{array}$} & \multirow{2}{*}{$\begin{array}{c}\text { Clay } \\
\% \\
\end{array}$} & \multirow{2}{*}{\multicolumn{2}{|c|}{$\begin{array}{c}\text { Bulk } \\
\text { Density } \\
\text { gm/cm3 }\end{array}$}} & & \\
\hline & & & & & & & & & \\
\hline 0-30 & \multicolumn{2}{|c|}{56.8} & 21.7 & 3.4 & 14.0 & \multicolumn{2}{|c|}{1.38} & 3.8 & Sandy \\
\hline $30-60$ & \multicolumn{2}{|c|}{56.5} & 28.6 & 2.0 & 10.0 & \multicolumn{2}{|c|}{1.41} & 4 & Sandy \\
\hline $60-90$ & \multicolumn{2}{|c|}{70.8} & 17.3 & 2.0 & 6.0 & \multirow{2}{*}{\multicolumn{2}{|c|}{$\begin{array}{l}1.4 \\
1.4\end{array}$}} & 3.5 & Sandy \\
\hline Mean & & 63 & 22.53 & 2.46 & 10 & & & 3.76 & Sandy \\
\hline \multicolumn{10}{|c|}{ (b): Chemical analysis } \\
\hline \multirow{3}{*}{$\begin{array}{c}\text { Depth } \\
\text { cm }\end{array}$} & \multicolumn{8}{|c|}{ Soluble ions (meq/100gm.soil) } & \multirow{3}{*}{$\begin{array}{c}\text { E.C. } \\
\text { mmhos/ } \\
\text { cm. at } 25 \mathrm{C}\end{array}$} \\
\hline & \multicolumn{4}{|c|}{ Cations } & & \multicolumn{3}{|c|}{ Anions } & \\
\hline & $\mathrm{Ca}^{++}$ & $\mathrm{Mg}^{++}$ & $\mathrm{Na}^{+}$ & $\mathrm{K}^{+}$ & $\mathrm{CO3}^{--}$ & $\mathrm{Hco3}^{-}$ & $\mathrm{Cl}^{-}$ & So ${ }^{--}$ & \\
\hline $0-30$ & 0.71 & 0.38 & 0.90 & 0.05 & trace & 0.35 & 0.85 & 0.92 & 0.66 \\
\hline $30-60$ & 0.26 & 0.06 & 0.42 & 0.02 & trace & 0.20 & 0.30 & 0.60 & 0.52 \\
\hline $60-90$ & 0.20 & 0.10 & 0.36 & 0.02 & trace & 0.20 & 0.30 & 0.54 & 0.40 \\
\hline Mean & 0.39 & 0.18 & 0.56 & 0.03 & trace & 0.25 & 0.483 & 0.686 & 0.526 \\
\hline
\end{tabular}

As such, the tested plants of banana $c v$. have a semilong pseudostem. For this purpose, only three new suckers per mat (stool) were selected from the second ratoon plants.The suckers of tested ratoon were nearly similar in height, growth and vigour. The experimental soil was classed as a sandy in texture and deficiency in fertility according to the mechanical and chemical analyses of samples (Table 1) desempled by (Jackson, 1958).

Accordingly, the experiment included 16 treatments each treatment comprised 3 replicates distributed in Randomized Complete Block Design (R.C.B.D).

The selected plants received all agricultural practices usually applied in banana plantation except $\mathrm{Zn}$ and $\mathrm{Fe}$ fertigation.

A factorial experiment with different rates of $\mathrm{Zn}$ and $\mathrm{Fe}$ and their combinations, four $\mathrm{ZnSO} 4$ fertigation rates $(0.0,5.4,10.8$ and $21.6 \mathrm{gm} / \mathrm{plant})$ and Fe (EDTA) (0.0, 9.0, 18.0 and $36 \mathrm{gm} /$ plant) were added every month during the period from April till October in both seasons.

The following parameters were used to evaluate the tested treatments:

\section{1-Vegetative growth:}

Morphological measurements were done at bunch shooting via the following parameters: Pseudostem height $(\mathrm{m})$; pseudostem circumference $(\mathrm{m})$, number of green leaves / plant and total green leaf area $\left(\mathrm{m}^{2}\right)$ which was calculated according to (Ahmed and Morsy, 1999) by using the formula:

$\mathbf{T L}=\mathbf{0 . 6 7}(\mathrm{I}+\mathrm{w})+\mathbf{1 0 7 . 1 5}$

Where: $\mathrm{T}=$ total, $\mathrm{L}=$ leaf, $\mathrm{I}=$ length, $\mathrm{w}=$ width.

\section{2-Flowering:}

The periods from sucker emergence to bunch shooting and from sucker emergence to bunch harvesting were calculated.

\section{3-Bunch characteristics:}

At time of harvesting the bunch weight in $\mathrm{Kg}$., average number of hands per bunch, average number of fingers per bunch, hand weight $(\mathrm{g})$, average number of fingers/hand and weight of finger $(\mathrm{g})$ were determined.

\section{4-Leaf mineral content:}

At bunch shooting, leaf samples were taken from the third upper most leaf in the succession of leaves from the plant top. Samples of fresh leaves were dried at $70 \mathrm{C}^{0}$ to a constant weight, then ground and subjected to the following determinations:

a) Total nitrogen was determined by using the microkjeldahl method as described by Pregl (1945)

b) Phosphorus was determined colorimetrically according to A.O.A.C. method (1985).

c) Potassium was determined using a flame photometer according to the method mentioned by Brown \& Lilleland (1946).

d) Zinc and iron were determined spectrophotometrically using an Atomic absorption according to Brandifeld and Spincer (1965)

$\mathrm{N}, \mathrm{P}$ and $\mathrm{K}$ were estimated as percentage of dry weight. Other elements $\mathrm{Zn}$ and $\mathrm{Fe}$ were estimated as $\mathrm{ppm} / \mathrm{g}$ dry weight.

The obtained data were subjected to analysis of variance for factorial design in randomized complete blocks with three replicates in each treatment (Snedecor\& Cochran, 1980). The means were compared 
by using the method of new least significant differences (New L.S.D. at o.05) described by Waller\& Duncan (1969).

\section{RESULTS AND DISCUSSION}

Data in Table (2) show that, vegetative growth (pseudostem height, pseudostem circumference, number of green leaves and leaf area) of the GrandNain banana at bunch shooting stage in both seasons significantly varied due to $\mathrm{Zn}$ - fertigation rates $(0.0,5.4,10.8$ and $21.6 \mathrm{~g} / \mathrm{plant})$ and Fe- fertigation rates $(0.0,9.0,18.0$ and $36.0 \mathrm{~g} / \mathrm{plant}$ ) and their combinations. The pseudostem height, circumference, number of green leaves/plant and leaf area were significantly increased by increasing $\mathrm{Zn}$ fertigation rate up to $21.6 \mathrm{~g} / \mathrm{plant}$ and this was true in the two seasons. Results of the effect of different $\mathrm{Zn}$ rates on vegetative growth of banana were in agreement with (Jordine, 1962), (Abdel- Kader,1986 and Abdel-Kader et al., 1992) ) and Moreira et al., 2007).

Table 2. Effect of $\mathrm{Zn}$ and Fe fertigation on vegetative growth of GrandNain banana plants during 2008/2009and2009/-2010

Pseudostem height (m)

\begin{tabular}{|c|c|c|c|c|c|c|c|c|c|c|}
\hline \multirow{3}{*}{$\begin{array}{c}\text { Zn- } \\
\text { fertigation } \\
\text { rates gm./ } \\
\text { plant }\end{array}$} & \multicolumn{5}{|c|}{ First season } & \multicolumn{5}{|c|}{ Second season } \\
\hline & \multicolumn{10}{|c|}{ Fe-fertigation rates gm./plant } \\
\hline & 0.0 & 9.0 & 18.0 & 36.0 & Av. & 0.0 & 9.0 & 18.0 & 36.0 & Av. \\
\hline $\mathbf{0 . 0}$ & 2.67 & 2.80 & 2.85 & 2.95 & 2.82 & 2.70 & 2.74 & 2.84 & 2.92 & 2.8 \\
\hline 5.4 & 3.00 & 3.10 & 3.15 & 3.17 & 3.10 & 2.75 & 2.87 & 2.92 & 2.98 & 2.88 \\
\hline 10.8 & 3.00 & 3.10 & 3.15 & 3.20 & 3.11 & 2.89 & 2.93 & 2.99 & 3.18 & 2.99 \\
\hline 21.6 & 3.10 & 3.20 & 3.25 & 3.30 & 3.21 & 3.00 & 3.10 & 3.19 & 3.20 & 3.12 \\
\hline Av. & 2.94 & 3.05 & 3.1 & 3.15 & & 2.83 & 2.91 & 2.98 & 3.07 & \\
\hline New L.S.D & $\mathrm{Zn}$ & $\mathrm{Fe}$ & \multirow{2}{*}{\multicolumn{2}{|c|}{$\begin{array}{c}\text { Interaction } \\
0.105\end{array}$}} & & $\mathrm{Zn}$ & $\mathrm{Fe}$ & \multirow{2}{*}{\multicolumn{2}{|c|}{$\begin{array}{c}\text { Interaction } \\
0.066\end{array}$}} & \\
\hline at 0.05 & 0.053 & 0.053 & & & & 0.043 & 0.043 & & & \\
\hline \multicolumn{11}{|c|}{ Pseudostem circumference (m) } \\
\hline $\mathbf{0 . 0}$ & 0.70 & 0.78 & 0.80 & 0.84 & 0.78 & 0.68 & 0.76 & 0.81 & 0.83 & 0.77 \\
\hline 5.4 & 0.72 & 0.76 & 0.77 & 0.82 & 0.77 & 0.76 & 0.77 & 0.79 & 0.83 & 0.79 \\
\hline 10.8 & 0.75 & 0.79 & 0.81 & 0.82 & 0.79 & 0.78 & 0.80 & 0.82 & 0.85 & 0.81 \\
\hline 21.6 & 0.81 & 0.84 & 0.85 & 0.87 & 0.84 & 0.82 & 0.86 & 0.87 & 0.88 & 0.86 \\
\hline Av. & 0.74 & 0.79 & 0.81 & 0.84 & & 0.76 & 0.80 & 0.82 & 0.85 & \\
\hline New L.S.D & & $\mathrm{Fe}$ & \multirow{2}{*}{\multicolumn{3}{|c|}{$\begin{array}{c}\text { Interaction } \\
0.047\end{array}$}} & $\mathrm{Zn}$ & $\mathrm{Fe}$ & \multirow{2}{*}{\multicolumn{2}{|c|}{$\begin{array}{l}\text { Interaction } \\
0.033\end{array}$}} & \\
\hline at 0.05 & 0.024 & 0.024 & & & & 0.017 & 0.017 & & & \\
\hline \multicolumn{11}{|c|}{ Number of green leaves/ plant } \\
\hline 0.0 & 11.0 & 12.0 & 13.0 & 13.0 & 12.25 & 11.3 & 12.0 & 13.0 & 13.0 & 12.32 \\
\hline 5.4 & 12.0 & 13.0 & 13.0 & 13.0 & 12.75 & 12.0 & 13.0 & 13.0 & 13.0 & 12.75 \\
\hline 10.8 & 13.0 & 13.0 & 13.0 & 13.0 & 13.00 & 13.0 & 13.3 & 13.3 & 13.3 & 13.22 \\
\hline 21.6 & 13.0 & 13.0 & 13.33 & 13.67 & 13.25 & 13.3 & 13.4 & 13.5 & 13.5 & 13.42 \\
\hline Av. & 12.25 & 12.75 & 13.08 & 13.17 & & 12.40 & 12.92 & 13.20 & 13.20 & \\
\hline $\begin{array}{l}\text { New L.S.D } \\
\text { at } 0.05\end{array}$ & $\begin{array}{l}\mathrm{Zn} \\
0.737\end{array}$ & $\begin{array}{l}\mathrm{Fe} \\
0.737\end{array}$ & \multicolumn{2}{|c|}{$\begin{array}{c}\text { Interaction } \\
1.473\end{array}$} & & $\begin{array}{l}\mathrm{Zn} \\
0.673\end{array}$ & $\begin{array}{c}\mathrm{Fe} \\
0.673\end{array}$ & \multicolumn{2}{|c|}{$\begin{array}{l}\text { Interaction } \\
1.346\end{array}$} & \\
\hline \multicolumn{11}{|c|}{ Leaf area/plant $\left(\mathrm{m}^{2}\right)$} \\
\hline 0.0 & 13.685 & 15.640 & 16.184 & 16.984 & 15.623 & 14.05 & 15.57 & 16.09 & 17.09 & 15.70 \\
\hline 5.4 & 16.320 & 16.617 & 17.636 & 19.225 & 17.449 & 16.43 & 16.52 & 17.74 & 19.15 & 17.46 \\
\hline 10.8 & 16.320 & 17.340 & 17.425 & 19.127 & 17.553 & 16.49 & 17.48 & 17.62 & 19.27 & 17.715 \\
\hline 21.6 & 16.184 & 16.979 & 19.380 & 22.950 & 18.873 & 16.38 & 17.08 & 19.48 & 20.35 & 18.322 \\
\hline Av. & 15.627 & 16.644 & 17.656 & 19.571 & & 15.837 & 16.662 & 17.732 & 18.965 & \\
\hline $\begin{array}{c}\text { New L.S.D } \\
\text { at } 0.05\end{array}$ & $\begin{array}{l}\mathrm{Zn} \\
1.005\end{array}$ & $\begin{array}{l}\mathrm{Fe} \\
1.005\end{array}$ & $\begin{array}{r}\text { Inte } \\
2 .\end{array}$ & $\begin{array}{l}\text { action } \\
10\end{array}$ & & $\begin{array}{l}\mathrm{Zn} \\
1.462\end{array}$ & $\begin{array}{l}\mathrm{Fe} \\
1.462\end{array}$ & $\begin{array}{l}\text { Inte } \\
2.9\end{array}$ & ction & \\
\hline
\end{tabular}

The data clarified obvious effect of Fe- fertigation on vegetative growth of the tested GrandNain banana plants. Vegetative growth was significantly increased by increasing Fe- fertigation rate up to $36.0 \mathrm{~g} / \mathrm{plant}$.

As for the previous available papers concerning the effect of Fe- fertigation on vegetative growth results of (Ziv, 1954), (Jordine, 1962) and (Abdel- Kader,1986, Hamam, 1988 and Abdel-Kader et al., 1992) working on banana are in harmony and support the obtained herein results.

The effect of interactions between $\mathrm{Zn}$ and $\mathrm{Fe}$ fertigation on vegetative growth are presented in Table (2). It is clear that, the interaction $(\mathrm{Zn} \times \mathrm{Fe})$ were statistically significant, which indicate a high degree of interdependence between the studied nutrients. This was apparent with all considered vegetative growth parameters. The highest values for vegetative growth in 
both seasons resulted from $21.6 \mathrm{~g} \mathrm{Zn}+36.0 \mathrm{~g}$ Fe. The beneficial effect of micro nutrients in enhancing vegetative growth was previously mentioned by Twyford (1967), Jordine (1962) and Ziv (1954).

Also, the importance of these minerals in the multibiological processes, like the role of $\mathrm{Zn}$ in the synthesis of IAA was reported by Nason (1950)

Data in Table (3) show that increasing the rate of $\mathrm{Zn}$ fertigation gradually and significantly shortened the time to flowering. Yet, the period to flowering is 411.0, $398.75,393.75,384.5$ and $414.5,424.25,423.25,401.0$ days in $0.0,5.4,10.8$ and $21.6 \mathrm{gm} /$ plant treatments respectively in both seasons.

With the effect of $\mathrm{Fe}$ - fertigation rates on the period to flowering, the uppermost tested rate $(36.0 \mathrm{gm} / \mathrm{plant})$ shortened the period to flowering than the other treatments (415.0 and 424.75 days against 398.75 and 415.75 days and 389.75 and 419.0 days) for $0.0,9.0$ and $18.0 \mathrm{gm} /$ plant in both seasons respectively. Interaction studies between the two main factors indicated that, the time to flowering was statistically significant. It was clear that, the treatment $(21.5 \mathrm{~g} . \mathrm{Zn}+36.0 \mathrm{~g} \mathrm{Fe} / \mathrm{plant})$ was the most effective in shortening the time to flowering in both seasons. The beneficial effect of micro nutrients in

Table3. Effect of $\mathrm{Zn}$ and Fe fertigation on time of flowering and time of harvesting over two crop cycles $(2008 / 2009-2009 / 2010)$

\begin{tabular}{|c|c|c|c|c|c|c|c|c|c|c|}
\hline \multirow{4}{*}{$\begin{array}{c}\text { Zn- } \\
\text { fertigation } \\
\text { rates gm./ } \\
\text { plant }\end{array}$} & \multicolumn{9}{|c|}{ Time to flowering (days) } & \\
\hline & \multicolumn{5}{|c|}{ First season } & \multicolumn{5}{|c|}{ Second season } \\
\hline & \multicolumn{10}{|c|}{ Fe-fertigation rates gm./plant } \\
\hline & 0.0 & 9.0 & 18.0 & 36.0 & Av. & 0.0 & 9.0 & 18.0 & 36.0 & Av. \\
\hline 0.0 & 425 & 425 & 404 & 390 & 411.0 & 432 & 415 & 416 & 395 & 414.5 \\
\hline 5.4 & 420 & 395 & 395 & 385 & 398.75 & 442 & 435 & 425 & 395 & 424.25 \\
\hline 10.8 & 420 & 390 & 380 & 385 & 393.75 & 430 & 423 & 420 & 420 & 423.25 \\
\hline 21.6 & 395 & 385 & 380 & 378 & 384.5 & 395 & 390 & 415 & 404 & 401.0 \\
\hline Av. & 415.0 & 398.75 & 389.75 & 384.50 & & 424.75 & 415.75 & 419.0 & 403.50 & \\
\hline $\begin{array}{c}\text { New L.S.D } \\
\text { at } 0.05 \\
\end{array}$ & $\begin{array}{l}\mathrm{Zn} \\
2.630 \\
\end{array}$ & $\begin{array}{l}\mathrm{Fe} \\
2.630\end{array}$ & \multicolumn{2}{|c|}{$\begin{array}{l}\text { Interaction } \\
5.262\end{array}$} & & $\begin{array}{l}\mathrm{Zn} \\
2.948 \\
\end{array}$ & $\begin{array}{l}\mathrm{Fe} \\
2.948\end{array}$ & \multicolumn{2}{|c|}{$\begin{array}{l}\text { Interaction } \\
5.896\end{array}$} & \\
\hline \multicolumn{11}{|c|}{ Time to harvesting (days) } \\
\hline \multirow{3}{*}{$\begin{array}{c}\mathrm{Zn}- \\
\text { fertigation } \\
\text { rates gm./ } \\
\text { plant } \\
\end{array}$} & \multicolumn{5}{|c|}{ First season } & \multicolumn{5}{|c|}{ Second season } \\
\hline & \multicolumn{10}{|c|}{ Fe-fertigation rates gm./plant } \\
\hline & $\mathbf{0 . 0}$ & 9.0 & 18.0 & 36.0 & Av. & $\mathbf{0 . 0}$ & 9.0 & 18.0 & 36.0 & Av. \\
\hline $\mathbf{0 . 0}$ & 515.0 & 520.0 & 511.0 & 542.0 & 509.5 & 568.0 & 560.0 & 535.0 & 515.0 & 544.0 \\
\hline 5.4 & 532.0 & 485.0 & 485.0 & 475.0 & 494.2 & 505.0 & 540.0 & 541.0 & 557.0 & 535.75 \\
\hline 10.8 & 510.0 & 490.0 & 475.0 & 475.0 & 487.5 & 540.0 & 548.0 & 530.0 & 530.0 & 537.0 \\
\hline 21.6 & 485.0 & 495.0 & 470.0 & 468.0 & 479.5 & 520.0 & 515.0 & 515.0 & 505.0 & 513.75 \\
\hline Av. & 510.5 & 497.5 & 485.25 & 490.0 & & 533.25 & 540.75 & 530.25 & 526.75 & \\
\hline $\begin{array}{c}\text { New L.S.D } \\
\text { at } 0.05\end{array}$ & $\begin{array}{l}\mathrm{Zn} \\
2.674\end{array}$ & $\begin{array}{l}\mathrm{Fe} \\
2.674\end{array}$ & \multicolumn{2}{|c|}{$\begin{array}{c}\text { Interaction } \\
5.348\end{array}$} & & $\begin{array}{l}\mathrm{Zn} \\
2.978\end{array}$ & $\begin{array}{l}\mathrm{Fe} \\
2.978\end{array}$ & \multicolumn{2}{|c|}{$\begin{array}{l}\text { Interaction } \\
5.956\end{array}$} & \\
\hline
\end{tabular}

regulating bunch shooting may be due to its role in improving the vegetative growth which in turn enhances the emergence of floral inflorescences as reported by Swidan (1972), who noticed that the differentiation of inflorescences in banana plants cv. Hindi is positively correlated with the total leaf area per plant.

Data in Table (3) showed that gradual increasing the rate of $\mathrm{Zn}$ - fertigation gradually and significantly shortened the time to harvesting. Yet, the period to harvesting is $509.5 ; 494.2 ; 487.5 ; 479.5$ and 544.0 ; $535.75 ; 537.0 ; 513.75$ days in $0.0,5.4,10.8$ and 21.5 $\mathrm{gm} /$ plant treatments respectively in both seasons. With the effect of $\mathrm{Fe}$ - fertigation rates on the period to harvestng, the uppermost tested rate $(36.0 \mathrm{gm} / \mathrm{plant})$ shortened the period to harvesting than the other treatment $(510.5,533.25$ days against 497.5, 540.75 days and 485.25, 530.25 days) for 0.0, 9.0 and 18.0 $\mathrm{gm} /$ plant treatments in both seasons respectively. Interaction studies between the two main factors concerning the time to harvesting were statistically significant. It was clear that, the treatment $(21.6 \mathrm{~g} . \mathrm{Zn}+$ $36.0 \mathrm{~g} \mathrm{Fe} /$ plant) was the effective in shortening the time to harvesting in both seasons. 
Data in Table (4) showed that, bunch characteristics (Bunch weight and average number of hand per bunch) significantly varied in response to $\mathrm{Zn}$ and $\mathrm{Fe}$ fertigation treatment. Bunch characteristics were significantly increased by increasing the $\mathrm{Zn}$-fertigation rate, the heaviest bunches were produced in plants received the high amount of $\mathrm{Zn}$ and Fe fertigation treatment (21.6g $\mathrm{Zn}+36.0 \mathrm{~g} / \mathrm{plant}$ ) and the lightest bunches were obtained from the plants without $\mathrm{Zn}$ and Fe fertigation $(0.0 \mathrm{gZn}+$ $0.0 \mathrm{~g} \mathrm{Fe} /$ plant)

As for number of hands/bunch, the greatest number of hands/bunch (12.0 and 13.2) and number of fingers/ bunch (240.20 and 197.9) in both seasons respectively were obtained from plants received the high amount of $\mathrm{Zn}$ and Fe fertigation.

Concerning weight of finger (Table 5) the heaviest finger was produced in plants received the high amount of $\mathrm{Zn}$ and $\mathrm{Fe}$ fertigation treatment $(21.6 \mathrm{~g} \mathrm{Zn+}$

Table 4. Effect of $\mathrm{Zn}$ and Fe fertigation on bunch characteristics of GrandNain banana plants during 2008/2009-2009/2010

\begin{tabular}{|c|c|c|c|c|c|c|c|c|c|c|}
\hline \multirow{4}{*}{$\begin{array}{c}\text { Zn- } \\
\text { fertigation } \\
\text { Rates gm./ } \\
\text { plant }\end{array}$} & \multicolumn{9}{|c|}{ Bunch weight/plant $(\mathbf{k g})$} & \\
\hline & \multicolumn{5}{|c|}{ First season } & \multicolumn{5}{|c|}{ Second season } \\
\hline & \multicolumn{10}{|c|}{ Fe-fertigation rates gm./plant } \\
\hline & 0.0 & 9.0 & 18.0 & 36.0 & Av. & 0.0 & 9.0 & 18.0 & 36.0 & Av. \\
\hline 0.0 & 23.83 & 28.70 & 29.90 & 35.65 & 29.52 & 23.90 & 27.70 & 29.56 & 34.50 & 28.91 \\
\hline 5.4 & 23.90 & 30.69 & 35.86 & 40.34 & 32.70 & 25.50 & 32.0 & 35.80 & 36.55 & 32.46 \\
\hline 10.8 & 25.17 & 34.66 & 35.87 & 42.77 & 34.62 & 26.80 & 30.50 & 36.33 & 38.70 & 33.08 \\
\hline 21.6 & 25.80 & 35.66 & 38.79 & 43.00 & 35.81 & 28.80 & 30.70 & 33.90 & 39.50 & 33.22 \\
\hline Av. & 24.67 & 32.43 & 35.10 & 40.44 & & 26.25 & 30.22 & 33.90 & 37.31 & \\
\hline $\begin{array}{c}\text { New L.S.D } \\
\text { at } 0.05\end{array}$ & $\begin{array}{l}\mathrm{Zn} \\
0.847\end{array}$ & $\begin{array}{l}\mathrm{Fe} \\
0.847\end{array}$ & \multicolumn{2}{|c|}{$\begin{array}{l}\text { Interaction } \\
1.694\end{array}$} & & $\begin{array}{l}\mathrm{Zn} \\
2.222\end{array}$ & $\begin{array}{l}\mathrm{Fe} \\
2.222\end{array}$ & \multicolumn{2}{|c|}{$\begin{array}{l}\text { Interaction } \\
4.444\end{array}$} & \\
\hline \multicolumn{11}{|c|}{ Average number of hands per bunch } \\
\hline $\mathbf{0 . 0}$ & 9.75 & 10.30 & 10.60 & 11.00 & 10.41 & 10.0 & 10.3 & 10.5 & 11.0 & 10.45 \\
\hline 5.4 & 10.00 & 10.33 & 11.00 & 11.00 & 10.58 & 10.6 & 10.9 & 11.0 & 11.0 & 10.87 \\
\hline 10.8 & 10.20 & 11.00 & 11.00 & 11.00 & 10.80 & 11.0 & 11.0 & 11.0 & 12.0 & 11.25 \\
\hline 21.6 & 10.86 & 11.00 & 12.00 & 12.00 & 11.46 & 11.3 & 11.8 & 12.6 & 13.2 & 12.22 \\
\hline Av. & 10.20 & 10.66 & 11.15 & 11.25 & & 10.72 & 11.0 & 11.27 & 11.8 & \\
\hline $\begin{array}{l}\text { New L.S.D } \\
\text { at } 0.05\end{array}$ & $\begin{array}{l}\mathrm{Zn} \\
1.208 \\
\end{array}$ & $\begin{array}{l}\mathrm{Fe} \\
1.208\end{array}$ & \multicolumn{2}{|c|}{$\begin{array}{l}\text { Interaction } \\
2.417\end{array}$} & & $\begin{array}{l}\mathrm{Zn} \\
0.939 \\
\end{array}$ & $\begin{array}{l}\mathrm{Fe} \\
0.939 \\
\end{array}$ & \multicolumn{2}{|c|}{$\begin{array}{l}\text { Interaction } \\
1.879\end{array}$} & \\
\hline \multicolumn{11}{|c|}{ Average number of fingers/bunch } \\
\hline $\mathbf{0 . 0}$ & 171.12 & 190.55 & 200.06 & 220.80 & 195.63 & 157.6 & 171.5 & 179.6 & 190.5 & 174.8 \\
\hline 5.4 & 190.28 & 209.60 & 231.67 & 220.03 & 212.89 & 166.9 & 177.6 & 180.7 & 194.3 & 179.87 \\
\hline $\mathbf{1 0 . 8}$ & 190.84 & 200.86 & 231.37 & 220.80 & 210.97 & 169.6 & 180.5 & 186.5 & 196.5 & 183.27 \\
\hline 21.6 & 200.20 & 220.00 & 220.50 & 240.20 & 220.22 & 172.3 & 182.5 & 193.3 & 197.9 & 186.5 \\
\hline Av. & 188.11 & 205.25 & 220.9 & 225.46 & & 166.6 & 178.0 & 185.0 & 194.8 & \\
\hline $\begin{array}{c}\text { New L.S.D } \\
\text { at } 0.05\end{array}$ & $\begin{array}{l}\mathrm{Zn} \\
2.545 \\
\end{array}$ & $\begin{array}{l}\mathrm{Fe} \\
2.545\end{array}$ & $\begin{array}{l}\text { Inter } \\
5.09\end{array}$ & ction & & $\begin{array}{l}\mathrm{Zn} \\
2.375 \\
\end{array}$ & $\begin{array}{l}\mathrm{Fe} \\
2.375 \\
\end{array}$ & & $\begin{array}{l}\text { raction } \\
50\end{array}$ & \\
\hline
\end{tabular}

$36.0 \mathrm{~g} / \mathrm{plant})$ and the lightest finger was obtained from the plants without $\mathrm{Zn}$ and Fe fertigation $(0.0 \mathrm{gZn}+0.0 \mathrm{~g}$ $\mathrm{Fe} /$ plant). Results of the effect of different $\mathrm{Zn}$ rates on the yield of banana were in harmony with the finding of (Abdel- Kader,1986; Abdel-Kader et al., 1992, Ghanta and Mitra 1993 and Sallam et al.,2002). Interaction between the two main factors concerning bunch characteristics was statistically significant which referred to $\mathrm{Zn}$ and $\mathrm{Fe}$ fertigation act dependently in this respect Table (4).

Data in Table (5) revealed that the heaviest weight of hands were produced in plants received the high amount of $\mathrm{Zn}$ and $\mathrm{Fe}$-fertigation treatment $(21.6 \mathrm{~g} . \mathrm{Zn}+$ $36.0 \mathrm{~g} . \mathrm{Fe} / \mathrm{plant}$ ) and the lightest hands were obtained form plants without fertigation with $\mathrm{Zn}$ and $\mathrm{Fe}$ fertigation. For example heaviest hand was $(2100.0 \mathrm{~g}$ and $2000.7 \mathrm{~g}$.) and the highest number of fingers/hand (20.20 and 21.3 ) respectively in both seasons. 
Table 5. Effect of $\mathrm{Zn}$ and Fe fertigation on hand characteristics of GrandNain banana plants during 2008/2009-2009/2010

\begin{tabular}{|c|c|c|c|c|c|c|c|c|c|c|}
\hline \multirow{4}{*}{$\begin{array}{c}\text { Zn- } \\
\text { fertigation } \\
\text { rates gm./ } \\
\text { plant } \\
\end{array}$} & \multicolumn{9}{|c|}{ Hand weight (g) } & \\
\hline & \multicolumn{5}{|c|}{ First season } & \multicolumn{5}{|c|}{ Second season } \\
\hline & \multicolumn{10}{|c|}{ Fe-fertigation rates gm./plant } \\
\hline & 0.0 & 9.0 & 18.0 & 36.0 & Av. & 0.0 & 9.0 & 18.0 & 36.0 & Av. \\
\hline $\mathbf{0 . 0}$ & 761.2 & 1461.70 & 1681.80 & 1827.50 & 1433.0 & 876.2 & 1450.5 & 1661.7 & 1807.3 & 1448.92 \\
\hline 5.4 & 1666.80 & 1674.30 & 1774.50 & 1779.30 & 1723.72 & 1665.7 & 1770.3 & 1773.5 & 1780.4 & 1747.47 \\
\hline $\mathbf{1 0 . 8}$ & 1695.40 & 1780.90 & 1801.10 & 2100.00 & 1844.35 & 1679.5 & 1779.9 & 1800.3 & 1900.7 & 1790.1 \\
\hline 21.6 & 1794.80 & 1837.00 & 2071.80 & 2100.00 & 1950.9 & 1799.5 & 1885.0 & 1970.8 & 2000.7 & 1914.0 \\
\hline Av. & 1479.55 & 1688.47 & 1832.3 & 1951.7 & & 1505.22 & 1721.42 & 1801.57 & 1872.27 & \\
\hline $\begin{array}{c}\text { New L.S.D } \\
\text { at } 0.05\end{array}$ & $\begin{array}{l}\mathrm{Zn} \\
27.682\end{array}$ & $\begin{array}{l}\mathrm{Fe} \\
27.682\end{array}$ & \multicolumn{2}{|c|}{$\begin{array}{l}\text { Interaction } \\
55.364\end{array}$} & & $\begin{array}{l}\mathrm{Zn} \\
34.600\end{array}$ & \multicolumn{2}{|c|}{$\begin{array}{l}\mathrm{Fe} \\
34.600 \\
\end{array}$} & $\begin{array}{l}\text { Interaction } \\
69.201\end{array}$ & \\
\hline \multicolumn{11}{|c|}{ Average number of fingers/hand } \\
\hline & 0.0 & 9.0 & 18.0 & 36.0 & Av. & 0.0 & 9.0 & 18.0 & 36.0 & Av. \\
\hline $\mathbf{0 . 0}$ & 19.00 & 19.28 & 20.20 & 20.20 & 19.67 & 19.0 & 19.5 & 20.0 & 20.5 & 19.75 \\
\hline 5.4 & 19.50 & 20.00 & 21.00 & 20.20 & 20.17 & 19.3 & 20.0 & 20.2 & 21.0 & 20.12 \\
\hline 10.8 & 19.20 & 20.44 & 20.50 & 21.28 & 20.35 & 19.5 & 20.0 & 20.3 & 20.5 & 20.07 \\
\hline 21.6 & 20.00 & 20.00 & 20.20 & 20.20 & 20.1 & 20.5 & 21.0 & 21.2 & 21.3 & 21.00 \\
\hline Av. & 19.42 & 19.93 & 20.47 & 20.47 & & 19.57 & 20.12 & 20.42 & 20.82 & \\
\hline $\begin{array}{c}\text { New L.S.D } \\
\text { at } 0.05 \\
\end{array}$ & $\begin{array}{l}\mathrm{Zn} \\
0.986 \\
\end{array}$ & $\begin{array}{l}\mathrm{Fe} \\
0.986 \\
\end{array}$ & \multicolumn{2}{|c|}{$\begin{array}{l}\text { Interaction } \\
1.972 \\
\end{array}$} & & $\begin{array}{l}\mathrm{Zn} \\
0.421 \\
\end{array}$ & $\begin{array}{l}\mathrm{Fe} \\
0.421 \\
\end{array}$ & \multicolumn{2}{|c|}{$\begin{array}{l}\text { Interaction } \\
0.842 \\
\end{array}$} & \\
\hline \multicolumn{11}{|c|}{ Weight of finger (g) } \\
\hline & 0.0 & 9.0 & 18.0 & 36.0 & Av. & 0.0 & 9.0 & 18.0 & 36.0 & Av. \\
\hline $\mathbf{0 . 0}$ & 19.00 & 19.28 & 20.20 & 20.20 & 19.67 & 65.2 & 71.5 & 77.6 & 89.0 & 75.82 \\
\hline 5.4 & 20.00 & 20.00 & 20.20 & 20.20 & 20.10 & 66.6 & 76.7 & 78.9 & 90.0 & 78.05 \\
\hline 10.8 & 19.50 & 20.00 & 21.00 & 20.20 & 20.17 & 75.9 & 79.7 & 82.5 & 95.0 & 83.27 \\
\hline 21.6 & 19.20 & 20.44 & 20.50 & 21.28 & 20.35 & 78.8 & 82.9 & 87.5 & 96.1 & 86.32 \\
\hline Av. & 19.42 & 19.93 & 20.47 & 20.47 & & 71.625 & 77.7 & 81.625 & 92.525 & \\
\hline $\begin{array}{c}\text { New L.S.D } \\
\text { at } 0.05\end{array}$ & $\begin{array}{l}\mathrm{Zn} \\
0.986\end{array}$ & $\begin{array}{l}\mathrm{Fe} \\
0.986\end{array}$ & & $\begin{array}{l}\text { teraction } \\
972\end{array}$ & & $\begin{array}{l}\mathrm{Zn} \\
0.421\end{array}$ & $\begin{array}{l}\mathrm{Fe} \\
0.421\end{array}$ & & $\begin{array}{l}\text { eraction } \\
52\end{array}$ & \\
\hline
\end{tabular}

Macro and micro elements were determined in the leaves of tested Grand Nain banana as affected by $\mathrm{Zn}$ and Fe- fertigation rates in two tested seasons of investigation (Table 6). The data in the Table showed that $\mathrm{N}$ - concentration in leaf of treated plants tended to increase by increasing rate of $\mathrm{N}$ - fertigation gradually in the two tested seasons. The highest values of $\mathrm{N}$ concentration in this concern were noticed with 21.6g.Zn./plant.

Data recorded in Table (6) showed clear increasing effect of Fe-fertigation on nitrogen concentration in banana leaves during two examination seasons.Nitrogen concentrations in leaves were increased by increasing $\mathrm{Fe}$ - fertigation rates. The effects of interactions between $\mathrm{Zn}$ and Fe-fertigation on leaf $\mathrm{N}$ content are presented in Table (6). It is clear that all the possible interactions were statistically significant which indicate a high degree of interdependence between the studied nutrients. The highest values for $\mathrm{N}$ concentration in two tested seasons resulted from $21.6 \mathrm{~g} . \mathrm{Zn}+36.0 \mathrm{~g} . \mathrm{Fe} / \mathrm{plant}$

The obtained data showed clear increase in leaf $\mathrm{P}$ concentration as $\mathrm{Zn}$ - fertigation treatment was increased; 21.6g.Zn gave the highest concentration than the other treatments. Regarding leaf $\mathrm{P}$ content in response to $\mathrm{Fe}-$ fertigation rate, $36.0 \mathrm{~g} . \mathrm{Fe} / \mathrm{plant}$ treatment gave the highest concentration as compared with other treatments in two seasons.

Leaf $\mathrm{K}$ content tends to increase with increasing rate of $\mathrm{Zn}$-fertigation.

For instance, K concentrations were 3.021, 3.157; $3.157,3.252 ; 3.265,3.347, \quad 3.358$ and $3.39 \%$ in treatments of $0.0,5.4,10.8,21.6 \mathrm{~g} . \mathrm{Zn} / \mathrm{plant}$ in two tested seasons. $\mathrm{K}$ concentrations in GrandNain leaves tend to increase as $\mathrm{Fe}$ - fertigation rates were increased. 
Table 6. Effect of $\mathrm{Zn}$ and Fe fertigation on leaf mineral content of GrandNain banana cultivar over two crop cycles (2008/2009-2009/2010)

Leaf $N$ content \%

\begin{tabular}{|c|c|c|c|c|c|c|c|c|c|c|}
\hline \multirow{3}{*}{$\begin{array}{c}\text { Zn- } \\
\text { fertigation } \\
\text { rates gm./ } \\
\text { plant }\end{array}$} & \multicolumn{5}{|c|}{ First season } & \multicolumn{5}{|c|}{ Second season } \\
\hline & \multicolumn{10}{|c|}{ Fe-fertigation rates gm./plant } \\
\hline & 0.0 & 9.0 & 18.0 & 36.0 & Av. & 0.0 & 9.0 & 18.0 & 36.0 & Av. \\
\hline 0.0 & 2.96 & 2.16 & 2.16 & 3.35 & 2.66 & 2.43 & 2.46 & 2.46 & 2.53 & 2.47 \\
\hline 5.4 & 3.35 & 3.35 & 3.54 & 3.54 & 3.45 & 2.53 & 2.53 & 2.56 & 2.60 & 2.55 \\
\hline 10.8 & 3.74 & 3.74 & 3.74 & 3.74 & 3.74 & 3.13 & 3.20 & 3.35 & 3.48 & 3.29 \\
\hline 21.6 & 3.93 & 3.93 & 3.93 & 3.93 & 3.93 & 3.66 & 3.76 & 3.80 & 3.91 & 3.78 \\
\hline Av. & 3.50 & 3.29 & 3.34 & 3.64 & & 2.94 & 2.99 & 3.04 & 3.13 & \\
\hline $\begin{array}{c}\text { New LSD } \\
\text { at } 0.05\end{array}$ & $\begin{array}{l}\mathrm{Zn} \\
0.21\end{array}$ & $\begin{array}{l}\mathrm{Fe} \\
0.20\end{array}$ & \multicolumn{2}{|c|}{$\begin{array}{c}\text { Interaction } \\
0.021 \\
\end{array}$} & & $\begin{array}{l}\mathrm{Zn} \\
0.48 \\
\end{array}$ & $\begin{array}{l}\mathrm{Fe} \\
0.21\end{array}$ & \multicolumn{2}{|c|}{$\begin{array}{c}\text { Interaction } \\
0.41 \\
\end{array}$} & \\
\hline \multicolumn{11}{|c|}{ Leaf $P$ content $\%$} \\
\hline $\mathbf{0 . 0}$ & 0.553 & 0.575 & 0.587 & 0.635 & 0.587 & 0.410 & 0.466 & 0.475 & 0.492 & 0.607 \\
\hline 5.4 & 0.658 & 0.677 & 0.711 & 0.712 & 0.689 & 0.520 & 0.630 & 0.670 & 0.741 & 0.812 \\
\hline 10.8 & 0.740 & 0.743 & 0.790 & 0.796 & 0.767 & 0.741 & 0.747 & 0.780 & 0.793 & 0.957 \\
\hline 21.6 & 0.797 & 0.833 & 1.275 & 1.880 & 1.196 & 0.799 & 0.800 & 0.988 & 1.321 & 1.276 \\
\hline Av. & 0.687 & 0.707 & 0.841 & 1.006 & & 0.810 & 0.617 & 0.661 & 0.728 & \\
\hline $\begin{array}{c}\text { New LSD } \\
\text { at } 0.05 \\
\end{array}$ & $\begin{array}{l}\mathrm{Zn} \\
0.014 \\
\end{array}$ & $\begin{array}{l}\mathrm{Fe} \\
0.01 \\
\end{array}$ & \multicolumn{2}{|c|}{$\begin{array}{c}\text { Interaction } \\
0.017\end{array}$} & & $\begin{array}{l}\mathrm{Zn} \\
0.011 \\
\end{array}$ & $\begin{array}{l}\mathrm{Fe} \\
0.01 \\
\end{array}$ & \multicolumn{2}{|c|}{$\begin{array}{c}\text { Interaction } \\
0.014\end{array}$} & \\
\hline \multicolumn{11}{|c|}{ Leaf $K$ content \% } \\
\hline $\mathbf{0 . 0}$ & 3.007 & 3.007 & 3.035 & 3.035 & 3.021 & 3.100 & 3.130 & 3.200 & 3.200 & 3.157 \\
\hline 5.4 & 3.064 & 3.150 & 3.208 & 3.208 & 3.157 & 3.130 & 3.200 & 3.320 & 3.360 & 3.252 \\
\hline 10.8 & 3.236 & 3.265 & 3.265 & 3.294 & 3.265 & 3.320 & 3.330 & 3.360 & 3.380 & 3.347 \\
\hline 21.6 & 3.323 & 3.351 & 3.380 & 3.380 & 3.358 & 3.380 & 3.380 & 3.390 & 3.410 & 3.390 \\
\hline Av. & 3.157 & 3.193 & 3.222 & 3.229 & & 3.232 & 3.260 & 3.317 & 3.337 & \\
\hline $\begin{array}{c}\text { New LSD } \\
\text { at } 0.05\end{array}$ & $\begin{array}{l}\mathrm{Zn} \\
0.21\end{array}$ & $\begin{array}{l}\mathrm{Fe} \\
0.18\end{array}$ & \multicolumn{2}{|c|}{$\begin{array}{c}\text { Interaction } \\
0.22\end{array}$} & & $\begin{array}{l}\mathrm{Zn} \\
0.15\end{array}$ & $\begin{array}{l}\mathrm{Fe} \\
0.20\end{array}$ & \multicolumn{2}{|c|}{$\begin{array}{c}\text { Interaction } \\
0.24\end{array}$} & \\
\hline \multicolumn{11}{|c|}{ Leaf $\mathrm{Zn}$ content \% } \\
\hline $\mathbf{0 . 0}$ & 10.820 & 10.995 & 11.610 & 11.955 & 11.345 & 10.33 & 10.44 & 10.56 & 10.66 & 11.08 \\
\hline 5.4 & 12.510 & 12.565 & 12.690 & 12.995 & 12.69 & 10.66 & 11.00 & 11.33 & 11.33 & 10.497 \\
\hline 10.8 & 13.065 & 13.255 & 13.315 & 13.315 & 13.237 & 13.33 & 14.00 & 14.00 & 14.33 & 13.915 \\
\hline 21.6 & 13.795 & 15.780 & 15.850 & 17.060 & 15.621 & 14.44 & 17.50 & 17.66 & 18.10 & 16.925 \\
\hline AV. & 12.547 & 13.149 & 13.366 & 13.831 & & 12.19 & 13.235 & 13.387 & 13.605 & \\
\hline $\begin{array}{c}\text { New LSD } \\
\text { at } 0.05\end{array}$ & $\begin{array}{l}\mathrm{Zn} \\
1.21 \\
\end{array}$ & $\begin{array}{l}\mathrm{Fe} \\
1.04 \\
\end{array}$ & $\begin{array}{r}\text { Inter } \\
1 \\
\end{array}$ & $\begin{array}{l}\text { raction } \\
1.34\end{array}$ & & $\begin{array}{l}\mathrm{Zn} \\
1.21 \\
\end{array}$ & $\begin{array}{l}\mathrm{Fe} \\
1.04 \\
\end{array}$ & Int & $\begin{array}{l}\text { eraction } \\
1.23 \\
\end{array}$ & \\
\hline \multicolumn{11}{|c|}{ Leaf Fe content ppm } \\
\hline 0.0 & 62.50 & 63.50 & 77.00 & 81.00 & 71.00 & 60.00 & 61.00 & 65.00 & 67.00 & 63.25 \\
\hline 5.4 & 71.50 & 74.75 & 80.50 & 84.60 & 77.84 & 61.00 & 65.00 & 74.00 & 73.00 & 68.25 \\
\hline 10.8 & 78.50 & 83.00 & 86.25 & 89.00 & 84.19 & 71.00 & 74.00 & 80.00 & 89.00 & 78.50 \\
\hline 21.6 & 81.25 & 86.25 & 89.50 & 94.25 & 87.81 & 81.00 & 86.00 & 89.00 & 94.00 & 87.50 \\
\hline Av. & 73.44 & 76.87 & 83.31 & 87.21 & & 68.25 & 71.5 & 77 & 80.75 & \\
\hline $\begin{array}{c}\text { New L.S.D } \\
\text { at } 0.05\end{array}$ & $\begin{array}{l}\mathrm{Zn} \\
4.11\end{array}$ & $\begin{array}{r}\mathrm{Fe} \\
3.71\end{array}$ & $\begin{array}{r}\text { Intera } \\
4.21\end{array}$ & action & & $\begin{array}{l}\mathrm{Zn} \\
4.21\end{array}$ & $\begin{array}{l}\mathrm{Fe} \\
2.91\end{array}$ & & $\begin{array}{l}\text { teraction } \\
.31\end{array}$ & \\
\hline $\begin{array}{l}\text { The highest } \\
\text { were noticed } \\
3.337 \% \text { ) resp } \\
\text { fertigation }\end{array}$ & $\begin{array}{l}\text { values of } \\
\text { ith } 36.0 \text { \& }\end{array}$ & $\begin{array}{l}\text {-concer } \\
\text { e/plant } \\
\text { owever, } \\
\text { ant) }\end{array}$ & ations in & $\begin{array}{l}\text { the leaves } \\
(3.229 \text { and } \\
\mathrm{e} \text { of } \mathrm{Fe}- \\
\text { the } \mathrm{K}\end{array}$ & $\begin{array}{l}\text { cor } \\
\text { res } \\
\text { dif } \\
\text { lea }\end{array}$ & $\begin{array}{l}\text { tration } \\
\text { tively. } \\
\text { nces be } \\
\text { content }\end{array}$ & $\begin{array}{l}\text { (3.193 an } \\
\text { Interaction } \\
\text { tween the } \\
\text { in response }\end{array}$ & $\begin{array}{l}\text { id } 3.26^{\circ} \\
\text { studies } \\
\text { two mai } \\
\text { to } \mathrm{Zn} \text { an }\end{array}$ & $\begin{array}{l}\text { o) in tw } \\
\text { showed } \\
\text { n factors } \\
\text { d Fe fertili }\end{array}$ & $\begin{array}{l}\text { o seasol } \\
\text { significa } \\
\text { concerni } \\
\text { zation }\end{array}$ \\
\hline
\end{tabular}


The tabulated data showed that $\mathrm{Zn}$ and $\mathrm{Fe}$ concentration in GrandNain banana leaves varied according to $\mathrm{Zn}$ and $\mathrm{Fe}$ fertigation. For example, leaf $\mathrm{Zn}$ and $\mathrm{Fe}$ content tended to increase with increasing rate of $\mathrm{Zn}$ - fertigation. Nevertheless, the low rate of $\mathrm{Zn}$ and $\mathrm{Fe}-$ fertigation $(5.4 \mathrm{~g} . \mathrm{Zn}$ and $9.0 \mathrm{~g}$.Fe /plant) gave the lowest values of $\mathrm{Zn}$ and $\mathrm{Fe}$ concentration in the leaves in the two seasons. It is clear that in the two tested season interactions $(\mathrm{ZnxFe})$ were statistically significant in affecting leaf $\mathrm{Zn}$ and $\mathrm{Fe}$ content. Analogical results in this respect were reported on banana plants by many investigators Abdel-Kader et al., 1992 and Ghanta and Mitra 1993.

\section{RE FERENCES}

Association of Official Analytical Chemists (1985): Official methods of Analysis. A.O.A.C $12^{\text {th }}$ Ed . Benjami.

Abdel-Kader, A. M. (1986). Physiological studies on Hindi banana cultivar. Ph. D. Thesis, Zagazig Univ., Egypt.

Abdel- Kader, A.M.M., F.M.B.El- Makhtoun and M.B. Bastawros (1992). Effect of micro-nutrients foliar application on the vegetative growth and yield of Hindi banana (M. Cavendeshii). Egypt. J. Agric. Res., 70 (2) 613-624.

Ahmed, F.F. and M.H. Morsy (1999). A new method for measurement leaf area in different fruit species. Minia J. Agric. Res.\& Develop., Vol.(19): 97-105.

Borges, A. L., S. O. Silva, R. C. Caldas and C.A.S. Ledo( 2006). Teores foliares de nutrients em genotipos de bananeira. Rev. Bras. Frut. 28:314-318.

Brandifeld, F. G. and Spincer (1965). Leaf analysis as a guide to plant crop.IV. Determination of manganese, zinc, iron and copper by atomic absorption sepectrocopy . J. Sci. Food Agric., 16:33-38.

Brown, J.D. and O. Lilliland (1946). Rapid determination of potassium and sodium in plant material and soil extracts by flame photometery . Proc.Amer. Soc. Hort. Sci., 48:341-346.

Brown, P.H.,I . Cakmak and Q. Zhang( 1993). Form and function of zinc in plants. In: Zinc in soil and plants. Ed. A. D. Robson. Kluwer Academic Publishers, Dordrecht. Pp.93-106.

Cakmak, I. (1988). Morphologische und physiologische veranderungen bei zinkmange pflangen. Dissert Fak. IIIAgrarwissensch. Idr Univ. Hohenheim. Werner B. (ed) In: Nutritional Disorders of Plants pp. 270.

Epstein, E. and A. J. Bloom ( 2005). Mineral nutrition of plants; principles and perspectives. Sinauer, Sunderland, pp. 400.
Ghanta, P. K. and S. K. Mitra (1993). Effect of micronutrients on growth, flowering, leaf nutrient content and yield of banana cv. Giant Governor. Crop Research (Hisar),6(2):284-287.

Hamam, M. S. (1988). The effect of gibberellin and some micro nutrients sprays on the yield and fruit quality in cavendish banana (Musa cavendishi). M. Sc. Thesis, Fac. Agric., Minia Univ.

Jackson, M. L.( 1958). Soil chemical Analysis. Constable and Co. Ltd. London p.498.

Jordine, C. G. (1962). Metal deficiencies in banana. Nature, 194:1160-1163.

Longnecker, N. E. and A. D. Robson (1993). Distribution and transport of zinc in plants. Zinc in soil and plants. Ed. A.D. Robson, Kluwer Academic Publishers, Dordrecht. Pp. 79-92.

Moreira, A., L.A.C. Moraes, J.C.R. Pereira and C. Castro (2007). Retranslocation of nutrients and zinc sulphate fertilization of banana plants in central Amazon. Journal of Applied Horticulture, 9(2):91-96.

Nason, A. (1950). Effect of Zinc in deficiency on the synthesis of tryptophan by Nemrospora extracts. Sceince.III: 12 .

Pregl, F. (1945). Quantitative inorganic analysis 4 th ED., Churchill L td., London, pp. 72-74.

Sallam, A.A.M., A.S. Hosam El-Din and N.E. Ashour (2002). Effect of spraying some microelements on yield and fruit quality of Hindy banana. J. Agric. Sci. Mansoura Univ., 27(8): 5497-5504.

Snedecor, G.W. and W. G. Cochran (1980): Statistical Methods. Seventh editions. Iowa State Univ. Pres, Ames Iowa, U.S.A.

Swidan, A. M. (1972). Studies on floral differentiation in banana plant and the effect of nitrogen fertilization on flowering, fruiting and fruit characteristics. Ph. D. Thesis, Fac. Agric.,Cairo Univ.,Egypt.

Twyford, I. T. (1967): Banana nutrition: A review of principles and practice. J. Sci. Food and Agric. 18: 177183.

Waller, R.A. and Duncan, D.B. (1969): A Bayes Rule for the Symmetric Multiple Comparison Problem," Journal of the American Statistical Association, 64, 1484 -1499, and (1972) "Corrigenda," 67, 253 -255.

Werner, B. (1992). Nutritional Disorders of Plants. Longman New York, 267:303-355.

Ziv, D. (1954). Chlorosis of bananas and other plants in Jordan Valley due to Fe deficiency. Hassadeh, 25: 190193. 


\section{الملخص العربي}

\section{تأثير التسميد بالزنك والحديد على نمو ومحصول الموز صنف جراندنان في الاراضى الرملية}

فريده عبد الحميد عبد ربة

في تخربة حقلية على نباتات الموز جراندنان (نباتات منتجة مـن (التزهير) وكذلك عددالايام اللازمة حتى الحصاد وقد كان الزنك له

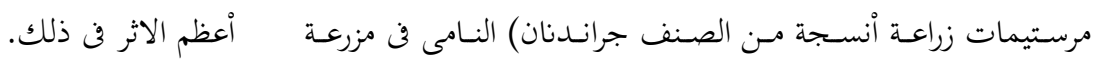

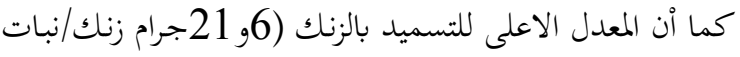

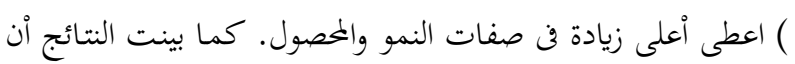

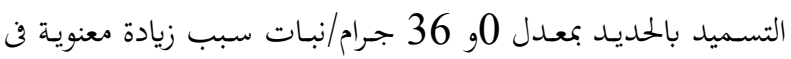

النمو وصفات السوباطات والاصابع. كما أن زيادة معدلات التسميد

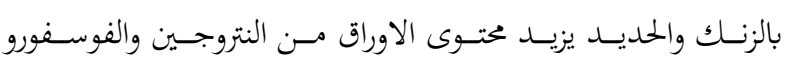

البوتاسيوم وكذلك الزنك والحديد.

وبناء" على نتائج الدراسة يمكن التوصية بالتسميد بالزنك والحديد

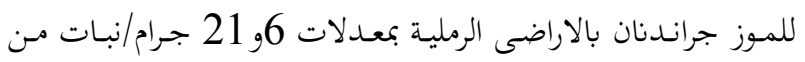

الزنك وكذلك أضافة 0و36 جرام/نبات مـن الحديد المخلبى شهريا"

ابتداء" من شهر أبريل - أْكتوبر.

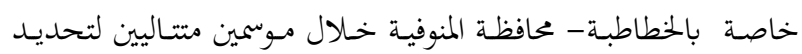

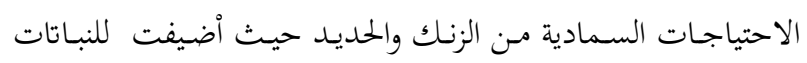
معدلات مختلفة من الزنك والحديد من خلال ماء الرى( حيث تروى

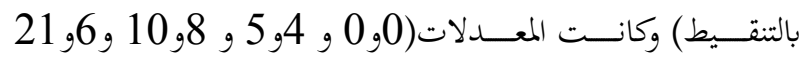

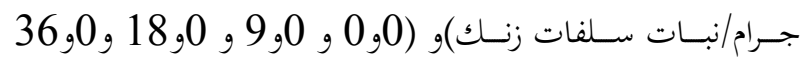

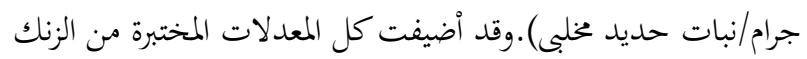

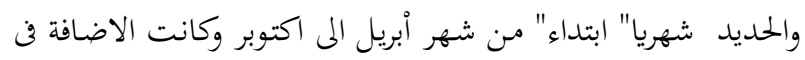

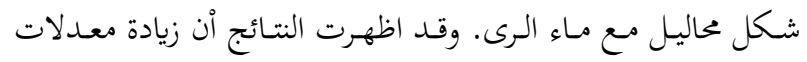

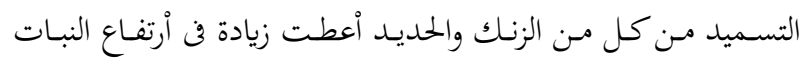

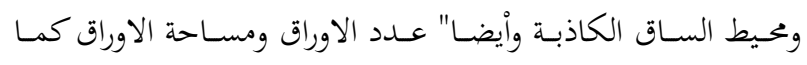
قللت عدد الايام اللازمة حتى مرحلة خروج النورة 